\title{
Uric Acid Predicts Long-Term Cardiovascular Risk in Type 2 Diabetes but Does Not Mediate the Benefits of Fenofibrate: the FIELD Study
}

\section{FIELD investigators}

2020-08

pÿFIELD investigators, Cao , J Y , Waldman , B , O Connell , R, Taskinen , M-R \& Keech , A C 2020 , ' Uric Acid Predicts Long-Term Cardiovascular Risk in Type 2 Diabetes but Does Not Mediate the Benefits of Fenofibrate: the FIELD Study ', Diabetes, obesity and metabolism , vol. 22 , no. 8 , pp. 1388-1396 . https://doi.org/10.1111/dom.14046

http://hdl.handle.net/10138/328675

https://doi.org/10.1111/dom.14046

unspecified

acceptedVersion

Downloaded from Helda, University of Helsinki institutional repository.

This is an electronic reprint of the original article.

This reprint may differ from the original in pagination and typographic detail.

Please cite the original version. 


\section{Uric Acid Predicts Long-Term Cardiovascular Risk in Type 2 Diabetes but Does Not Mediate the Benefits of Fenofibrate: the FIELD Study}

JY Cao ${ }^{1,2}$, B Waldman ${ }^{1,2}$, R O'Connell ${ }^{1}$, DR Sullivan ${ }^{1,2}$, RS Scott $^{3}, \mathrm{~N}^{\prime}$ Aryal $^{1}, \mathrm{~V}$ Gebski $^{1}$, I Marschner ${ }^{1}$, MR Taskinen ${ }^{4}$, RJ Simes ${ }^{1,2}$, N McGill ${ }^{1,2}$, AJ Jenkins ${ }^{1,2}$, AC Keech $^{1,2}$ on behalf of the FIELD investigators.

${ }^{1}$ National Health and Medical Research Council Clinical Trials Centre, Sydney, Australia

${ }^{2}$ Sydney Medical School, Sydney, Australia

${ }^{3}$ Lipid \& Diabetes Research Group, Christchurch Hospital, Christchurch, New Zealand

${ }^{4}$ Heart and Lung Centre, Cardiovascular Research Unit, Helsinki University Central Hospital, Helsinki, Finland

Running Title - Uric acid predicts cardiovascular disease in diabetes

\section{Correspondence Author}

Professor Anthony Keech

Department of Cardiology, Royal Prince Alfred Hospital, Camperdown, NSW, Australia, 2050

P: +61295625000

F: +61295625387

E: Tony@ctc.usyd.edu.au

This article has been accepted for publication and undergone full peer review but has not been through the copyediting, typesetting, pagination and proofreading process which may lead to differences between this version and the Version of Record. Please cite this article as doi: 


\section{Abstract}

Aims. Fenofibrate may lower long-term cardiovascular events. The biological mediators of its mechanisms of action are not fully understood. The current study aimed to determine whether the cardioprotective effects of fenofibrate are partly mediated through its uric acid (UA) lowering effects.

Methods. Data from the Fenofibrate Intervention and Event Lowering in Diabetes (FIELD) trial was utilised, comprising 9795 adults with type 2 diabetes (T2D) randomly allocated to treatment with fenofibrate or matching placebo. Plasma UA was measured before and after a 6-week active fenofibrate run-in phase in all participants. Cox proportional hazards models were used to explore the relationships between baseline UA, pre-to-post run-in reductions in UA and long-term cardiovascular outcomes.

Results. Mean baseline plasma UA was $0.33 \mathrm{mmol} / \mathrm{L}$ (SD 0.08). Baseline UA was a significant predictor of long-term cardiovascular events with every $0.1 \mathrm{mmol} / \mathrm{L}$ higher UA conferring a 21\% increase in event rate (HR 1.21, 95\% Cl 1.13-1.29, $p<0.001)$. This remained significant after adjustment for treatment allocation, cardiovascular risk factors, and renal function. The extent of UA reduction during fenofibrate run-in was also significant predictor of long-term cardiovascular events, with every $0.1 \mathrm{mmol} / \mathrm{L}$ greater reduction conferring a 14\% lower long-term risk (HR 0.86, 95\% Cl 0.76-0.97, $p=0.015)$. This effect was not modified by treatment allocation $\left(p_{\text {interaction }}=0.77\right)$.

Conclusions. UA is a strong independent predictor of long-term cardiovascular risk in adults with T2D. Although greater reduction in UA on fenofibrate is predictive of 
lower cardiovascular risk, this does not seem to mediate the cardioprotective effects of fenofibrate. 


\section{Introduction}

The degree of dyslipidaemia in type 2 diabetes (T2D) is closely linked to the risk of cardiovascular disease ${ }^{1}$.. The Fenofibrate Intervention and Event Lowering in Diabetes (FIELD) trial demonstrated reduction in total cardiovascular disease events with fenofibrate compared to matching placebo in patients with type 2 diabetes over a median 5 years follow up ${ }^{2}$. However, the mechanisms by which fenofibrate lowers cardiovascular event are not fully elucidated. Fibrates seem to have both lipoprotein related and non-lipoprotein related effects ${ }^{3}$. Previous statin trials have demonstrated positive relationships between the extent of low-density lipoprotein (LDL) cholesterol lowering and reduction in cardiovascular events ${ }^{4}$. In contrast, despite fenofibrate reducing triglyceride and increasing high-density lipoprotein (HDL) cholesterol levels, there are only weak correlations between improvements in lipid parameters and longterm clinical cardiovascular benefits, thereby prompting ongoing search for biological mediators.

Amongst the lipid-lowering agents, fenofibrate uniquely lowers circulating uric acid (UA) levels through increased renal clearance ${ }^{5}$, and has been shown to be potentially a powerful adjunct in the treatment of gout ${ }^{6}$. Previous post hoc analysis of the FIELD trial demonstrated that fenofibrate efficiently lowers UA levels during the prerandomisation 6-week fenofibrate run-in phase, and maintains this level in those subsequently allocated to fenofibrate vs. placebo ${ }^{6}$. Therefore, short-term change in UA levels is a reliable marker of long-term fenofibrate effects, and can be used to 
investigate the potential mediating role of UA in fenofibrate-related cardiovascular benefits.

In at least two large epidemiological studies, circulating UA levels have been reported to be a predictor of long-term cardiovascular events, independent of traditional risk factors 7,8 , although such results have not been replicated in other studies ${ }^{9,10}$. Additionally, the predictive ability of UA in those at high risk, such as patients with T2D, has not been reported.

The current post hoc analysis of the FIELD trial had two aims. Firstly, we aimed to explore the relationship between baseline UA levels and long-term cardiovascular events in adults with T2D. Secondly, we aimed to examine whether the cardiovascular benefits of fenofibrate are mediated through its UA lowering properties.

\section{Methods}

\section{Study Design and Participants}

The FIELD trial was a randomised double-blind placebo-controlled trial of fenofibrate in adults with T2D. The trial protocol and primary results have been previously published $^{2,11}$ (CONSORT Flow Diagram in Appendix 1). Briefly, patients with T2D aged between $50-75$ years were screened. To qualify for study entry, patients were required to have a baseline plasma total-cholesterol concentration of between 3.0 $\mathrm{mmol} / \mathrm{L}$ to $6.5 \mathrm{mmol} / \mathrm{L}$, plus either a total/HDL cholesterol ratio of 4.0 or more or a 
plasma triglyceride concentration of between $1.0 \mathrm{mmol} / \mathrm{L}$ to $5.0 \mathrm{mmol} / \mathrm{L}$. Patients were excluded on the basis of 1) being on or having indications for lipid-lowering therapy at study entry; 2) renal impairment (plasma creatinine $\geq 130 \mu \mathrm{mol} / \mathrm{L}$ ); 3 ) chronic liver disease or symptomatic gallbladder disease; and 4) cardiovascular event 3 months prior to recruitment.

All participants provided written informed consent, and the FIELD study protocol was approved by local and national ethics committees in accordance with the Declaration of Helsinki and Good Clinical Practice guidelines.

\section{Procedures}

All recruited participants underwent a 16-week run-in phase consisting of in order four weeks of lifestyle and dietary modifications, six weeks of single-blind placebo run-in and six weeks of single-blind fenofibrate run-in. The participants were subsequently randomised via a central computer, with stratification for important prognostic variables, into receiving either 200 mg daily of co-micronised fenofibrate or matching placebo. The participants were followed up for a median five years after randomisation.

Plasma UA was measured immediately prior to fenofibrate run-in (baseline) and prior to randomisation. Short-term change in UA with fenofibrate was calculated by taking the difference between the baseline and pre-randomisation levels. Additionally, a random cohort of 2,000 subjects was selected for a third measurement one year after randomisation. All plasma UA concentrations were measured in stored samples after 
study closure in a single central laboratory using a Cobas uricase enzymatic analyser (Roche, Mannheim).

The primary results of the FIELD trial have previously been published ${ }^{2}$. Briefly, fenofibrate treatment compared to matching placebo did not reduce the primary endpoint (first myocardial infarction or coronary heart disease death combined), but did reduce the prespecified secondary endpoint - total cardiovascular disease events (HR 0.89, 95\% Cl 0.80-0.99, $p=0.035)$. Total cardiovascular events were used as the outcome of interest for the present analyses to maximise the event rate. This was a composite of cardiovascular death, myocardial infarction, stroke, and coronary and carotid revascularisation.

Other covariates of interest include treatment allocation, gender, age, prior cardiovascular disease (history of myocardial infarction, angina, coronary revascularisation, stroke, claudication, peripheral vascular disease or peripheral revascularisation), smoking status, renal function and baseline body mass index (BMI), systolic blood pressure, HbA1c and non-HDL cholesterol. Renal function was explored using the estimated glomerular filtration rate (eGFR), urinary albumin-tocreatinine ratio and a combination thereof (eGFR $\geq$ or $<60 \mathrm{~mL} / \mathrm{min}$ and presence or absence of albuminuria). All other variables were defined in accordance to the original FIELD trial ${ }^{2}$.

\section{Statistical Analysis}


Baseline UA levels were divided into gender-specific tertiles. Categorical or continuous variables were compared between tertiles using appropriate univariate trend tests including linear-by-linear test and one-way ANOVA with linear contrast. The baseline UA and cumulative cardiovascular event frequency over median five year follow-up were also presented according to the number of metabolic syndrome components as defined by the Adult Treatment Panel III ${ }^{12}$. Furthermore, baseline UA level and prevalence of metabolic syndrome and its components were presented according to quintiles of cardiovascular risk using a previously derived model based on the FIELD data (Appendix 2).

Survival analysis with Kaplan-Meier curves and log-rank test was conducted comparing long-term cardiovascular events between the baseline UA tertiles. Cox proportional hazards model was used to assess the changes in the risk of cardiovascular events for every $0.1 \mathrm{mmol} / \mathrm{L}$ higher baseline UA. The model was further adjusted for traditional cardiovascular risk factors at baseline including treatment allocation, age, gender, smoking, systolic blood pressure, HbA1c, triglyceride, nonHDL cholesterol, prior cardiovascular disease and renal function. Subgroup analyses by important baseline prognostic factors were also undertaken to explore potential modifiers of the relationship between baseline UA and long-term cardiovascular events.

Cox proportional hazards model was used to assess the relationship between reduction in UA levels before and after the 6-week active fenofibrate run-in and longterm cardiovascular events, adjusting for randomised treatment allocation and 
baseline UA levels. To explore drivers of this relationship, post hoc analyses were performed by sequentially adjusting one variable (e.g., gender, renal function, HbA1c etc.) further to the model above.

Analyses of the relationships between total cardiovascular events and baseline and short-term change in UA levels were performed in accordance with an a priori statistical analyses plan. A two-sided $p$ value was considered significant without adjustment for multiple testing. Initial analyses (by JYC) using SPSS 23 (IBM, IL, USA) and confirmed independently (RO'C and NA) using SAS 9.4 (SAS Institute, NC, USA). The FIELD study is registered with ISRCTN, number ISRCTN64783481.

\section{Results}

Of the 9795 participants who underwent randomisation to either fenofibrate or matching placebo, baseline UA data were available for 9622 participants $(n=4807$ fenofibrate, $n=4815$ placebo).

Baseline UA levels ranged $0.11-0.79 \mathrm{mmol} / \mathrm{L}$ for the entire cohort (mean $0.33 \mathrm{mmol}$, SD $0.08 \mathrm{mmol} / \mathrm{L}$; Fig. 1). The baseline level for males was higher than for females (male: range $0.12-0.79 \mathrm{mmol} / \mathrm{L}$, mean $0.34 \mathrm{mmol} / \mathrm{L}$, SD $0.076 \mathrm{mmol} / \mathrm{L}$; female: range 0.11-0.71 mmol/L, mean $0.32 \mathrm{mmol} / \mathrm{L}$, SD $0.079 \mathrm{mmol} / \mathrm{L} ; p<0.001)$. The genderspecific thresholds for lowest-to-middle, and middle-to-highest tertile were 0.30 and 
$0.36 \mathrm{mmol} / \mathrm{L}$ respectively in males, and $0.27 \mathrm{mmol} / \mathrm{L}$ and $0.34 \mathrm{mmol} / \mathrm{L}$ respectively in females.

Baseline cardiovascular risk factors and cardiovascular disease were more prevalent in the highest UA tertile compared to the middle and lowest UA tertiles (Table 1). History of cardiovascular disease was noted in $24 \%$ of participants in the highest tertile, compared to $20 \%$ in the middle and lowest tertiles $(p<0.001)$. Dyslipidaemia, defined as low HDL-C (male: < $1.03 \mathrm{mmol} / \mathrm{L}$; female: < $1.29 \mathrm{mmol} / \mathrm{L}$ ) and high triglyceride ( $\geq 1.7 \mathrm{mmol} / \mathrm{L}$ ), was seen in $45 \%$ of participants in the highest tertile compared to $39 \%$ in the middle tertile and $30 \%$ in the lowest tertile $(p<0.001)$. Accordingly, triglyceride levels were highest in the highest UA tertile and lowest in the lowest UA tertile $(p<0.001)$. HbA1c had an inverse relationship to baseline UA levels, whereby $\mathrm{HbA1c}$ was the lowest in the highest UA tertile (6.6\%) and highest in the lowest UA tertile $(7.3 \%)(p<0.001)$. Baseline UA increased with the number of metabolic syndrome components $\left(p_{\text {trend }}<0.001 ;\right.$ Appendix 3 ). When stratified by quintiles of five-year cardiovascular risk, there was a linear increase in the prevalence of hypertension, high triglyceride, low HDL-C, and metabolic syndrome status, as well as baseline UA levels ( $p_{\text {trend }}<0.001$ in all cases; Appendix 4). Allopurinol usage was the highest in the lowest UA tertile, while diuretic usage was highest in the highest UA tertile. This likely reflects inherent UA-related lowering and elevating effects of allopurinol and of diuretics respectively. Use of these drugs did not modify the relationship between baseline uric acid and cardiovascular disease (allopurinol: $p_{\text {interaction }}=0.27$; diuretics: $p_{\text {interaction }}=0.39$ ) . 


\section{Baseline Uric Acid and Cardiovascular Events}

There were 395 (12.2\%), 428 (13.3\%) and 453 (14.3\%) onstudy cardiovascular events in the lowest, middle and highest baseline UA tertiles respectively over median 5 year follow up. As shown in Figure 3, Kaplan-Meier analysis revealed significant differences in rates of cardiovascular events (log rank: $\left.x^{2}=6.64, p=0.036\right)$, by baseline UA levels (Fig. 2).

Cox-proportional hazards model demonstrated a $21 \%$ increase in cardiovascular events with every $0.1 \mathrm{mmol} / \mathrm{L}$ higher baseline UA (HR 1.21, 95\% Cl 1.13-1.29, $p<0.001 ;$ Table 2). This corresponded to $16 \%$ change in risk for every one standard deviation change in UA levels, or $30 \%$ increase in risk from the median of the lowest UA tertile to the median of the highest tertile. Similar risks were seen by different periods of follow up (1-year: HR 1.19, 95\% Cl 1.01-1.40, $p=0.039$; 3-year: HR 1.20,

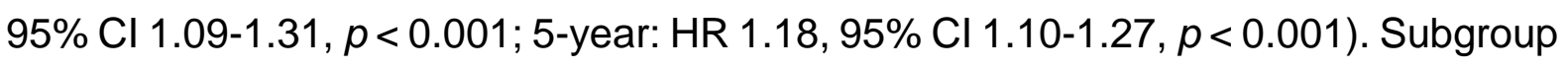
analyses revealed that higher baseline UA levels were associated with a smaller increase in on-trial cardiovascular events in those with systolic BP>140 mmHg or nonHDL-cholesterol over median than otherwise $\left(p_{\text {interaction }}=0.04\right.$ and 0.03 respectively; Appendix 5). No other interactions were noted between baseline UA and important prognostic variables on cardiovascular events.

Baseline UA levels were associated with multiple baseline cardiovascular prognostic variables (Appendix 6). After adjustment for these variables, baseline UA remained a significant predictor of long-term cardiovascular events, with every $0.1 \mathrm{mmol} / \mathrm{L}$ higher 
UA level conferring a 9\% increased risk (Table 2). On subgroup analysis of only participants not taking allopurinol or diuretics at baseline, UA remained a significant predictor of long-term cardiovascular disease (HR 1.17, 95\% CI 1.08-1.27, $p<0.001$ ).

\section{Short-Term Change in Uric Acid and Cardiovascular Events}

Data for short-term change in UA with 6-week single-blind fenofibrate run-in were available in 9347 participants (Appendix 7). A mean decrease in UA of $0.07 \mathrm{mmol} / \mathrm{L}$ (SD 0.05) was noted, corresponding to a $22 \%$ decrease from pre-run-in to prerandomisation level. The reduction in UA on fenofibrate was greater in females than males (female: mean 0.073 mmol/L, SD 0.05; male: mean 0.067 mmol/L, SD 0.05; $p$ $<0.001)$

The effect of long-term fenofibrate treatment on long-term cardiovascular events was analysed according to the extent of UA reduction during run-in as being above or below the median $(0.07 \mathrm{mmol} / \mathrm{L})$. This was undertaken on the rationale that if $U A$ reduction mediated the cardiovascular effects of fenofibrate, then those experiencing a greater reduction in UA during run-in would also derive a greater cardiovascular benefit on fenofibrate in the long-term (i.e. a lower HR). This was not observed - the cardiovascular effect of fenofibrate was comparable between those experiencing a greater and lesser UA reduction during run-in $\left(p_{\text {interaction }}=0.53\right.$, Fig. $\left.3 \mathrm{~A}\right)$

Adjusted for treatment allocation and baseline UA level, the short-term fenofibrateinduced change in UA was significantly associated with long-term cardiovascular events, with every $0.1 \mathrm{mmol} / \mathrm{L}$ greater reduction conferring a 14\% lower long-term risk 
(HR 0.86, 95\% Cl 0.76-0.97, $p=0.015$; Fig. 3B). There was no interaction between short-term change in UA levels on fenofibrate and treatment allocation with regards to long-term cardiovascular events $\left(p_{\text {interaction }}=0.77\right)$. This was confirmed by subgroup analyses by treatment allocation showing short-term change in UA levels was associated with on-trial cardiovascular events both in the fenofibrate (HR 0.87, 95\% $\mathrm{Cl} 0.73-1.04, p=0.12)$ and matching placebo groups (HR 0.85, 95\% $\mathrm{Cl} 0.72-1.01, p$ $=0.06)$, although neither was statistically significant. Post hoc analyses with additional variable adjustment to above model did not indicate modification of the relationship between short-term change in UA and long-term cardiovascular risk except by gender (Appendix 8). Notably, short-term reduction in UA was no longer associated with ontrial cardiovascular events after adjusting for baseline UA levels, treatment allocation and gender ( $\mathrm{HR} 0.94,95 \% \mathrm{Cl} 0.83-1.07, p=0.33)$. When applied to each gender separately, a trend was identified between baseline UA levels and long-term cardiovascular events in females (HR 0.78, 95\% Cl 0.61-1.01, $p=0.05)$, but not in males (HR 1.00, 95\% Cl 0.86-1.15, $p=0.95)$, although the interaction term was not significant $\left(p_{\text {interaction }}=0.10\right)$.

Change in UA levels during the active fenofibrate run-in period associated significantly with various baseline and other run-in change variables, although the strength of association was stronger with the baseline UA levels (Appendix 9). This was confirmed on multi-variable linear regression showing a $0.032 \mathrm{mmol} / \mathrm{L}$ higher reduction in UA levels with every 1 SD higher baseline UA ( $p<0.001$; Appendix 10). 


\section{Discussion}

UA is an emerging risk factor for cardiovascular disease in many, but not all, populations ${ }^{7,8,13}$, and is established as a component of the metabolic syndrome. However, the strength and relationship between UA levels and cardiovascular risk factors, in people with T2D is not fully elucidated. Various studies report linear, curvilinear and even U-shaped relationships between UA levels and chronic diabetes complications ${ }^{14,15}$. The current very large study in the robust FIELD trial of wellcharacterised adults with Type 2 diabetes demonstrated that baseline plasma UA levels are independently associated with on-trial cardiovascular events, with every 0.1 $\mathrm{mmol} / \mathrm{L}$ higher UA level predicting a $21 \%$ increase in risk. UA levels were related to the number of components of the metabolic syndrome. This relationship remained statistically significant after adjusting for traditional risk factors and renal function. The current study further explored whether the cardiovascular benefits of fenofibrate beyond its lipid effects are mediated at least partly through its UA-lowering properties. Short-term (6-weeks) exposure to fenofibrate reduced UA levels and was inversely associated with on-trial cardiovascular events i.e. those with greater UA reductions had lower event rates.

High levels of UA, the end-product of purine catabolism are strongly linked with gout and nephrolithiasis. Its links with cardiometabolic diseases, including obesity, hypertension ${ }^{16}$, diabetes ${ }^{17-19}$, metabolic syndrome ${ }^{20,21}$, heart failure ${ }^{22-24}$ and ischaemic heart disease ${ }^{25}$ are recognised, but underlying mechanisms are not wellestablished. The First National Health and Nutrition Examination Survey found that in 
nearly 6,000 subjects followed up for a mean 16 years, baseline UA independently predicted ischaemic heart disease mortality and cardiovascular mortality ${ }^{7}$. Notably, however, only $2 \%$ of the cohort had T2D at baseline. Studies examining the relationship between UA levels and cardiovascular risk exclusively in patients with T2D have yielded contrasting results ${ }^{26-28}$. This is likely a product of limited cohort size (approximately 1200-2700 patients) and between-study differences in the method of adjustment for important baseline risk factors, including gender, glycaemic control and renal function. A meta-analysis of $\mathrm{T} 2 \mathrm{D}$ studies demonstrated a small but statistically significant $28 \%$ increase in risk of macrovascular complications per $0.1 \mathrm{mmol} / \mathrm{L}$ higher UA levels in 20,981 patients with T2D ${ }^{29}$. However, the net effect was driven by peripheral vascular disease rather than cerebrovascular or coronary heart disease.

The current analysis to our knowledge is the largest exploration of the relationship between UA and cardiovascular outcomes in patients with T2D. At baseline, history of cardiovascular disease and presence of cardiovascular risk factors were shown to be most prevalent in the highest baseline UA tertile, with the only exception being HbA1c, which had an inverse relationship to UA levels. This relationship has been reported in various large epidemiological studies, showing that serum UA levels rise with glucose levels to a certain point, then begin to decrease with worsening glycaemia.

On-study cardiovascular events was also associated with higher baseline UA levels, even after adjustment for multiple clinical and biochemical risk factors, including 
baseline renal function and $\mathrm{HbA1c}$. The magnitude of the relationship was potentially modified by baseline systolic blood pressure and non-HDL cholesterol levels, whereby in those with worse hypertension and higher non-HDL cholesterol, baseline UA was less strongly (but still significantly) associated with subsequent cardiovascular events. However, this should be taken in the context of lack of statistical adjustment for multiple testing. Even though UA levels are usually higher in men than women, previous epidemiological studies have identified a stronger association between UA and subsequent cardiovascular events in women than men ${ }^{7}$. The current analysis did not identify any differences between the two genders ( $p$ for interaction effect $=0.5$ ). This may be influenced by our FIELD female participants predominantly being postmenopausal, and that in T2D, the female gender-related protection against cardiovascular disease is lost ${ }^{30}$.

The FIELD trial demonstrated an $11 \%$ reduction in the prespecified secondary outcome of total cardiovascular events in the fenofibrate vs. placebo group ${ }^{2}$. Fenofibrate has been shown to have non-lipid related effects on inflammatory and thrombotic processes ${ }^{31}$, one which is increased renal clearance of UA. The current study found that long-term fenofibrate cardiovascular benefits did not differ between those experiencing greater and lesser UA reductions during fenofibrate run-in ( $p$ for interaction effect $=0.53$ ), suggesting that UA lowering does not mediate the fenofibrate cardiovascular benefits. This was confirmed by the fact that short-term fenofibrateinduced reduction in UA during active run-in was associated with long-term cardiovascular benefits, although this relationship did not differ between those 
subsequently allocated to long-term fenofibrate or control $(p$ for interaction effect $=$ 0.77). Notably, in those who were allocated to long-term fenofibrate, the mean UA level at one year post-randomisation was similar to the post-run-in level ${ }^{6}$, meaning that the run-in change is an adequate surrogate for long-term change. Similar findings were noted in the Greek Atorvastatin and Coronary Heart Disease Evaluation Study, where a $5 \%$ reduction in UA over a mean 3-year follow up predicted a $15 \%$ reduction in risk of vascular events in those with metabolic syndrome and coronary artery disease. It is unclear, however, if the cardioprotective benefits of atorvastatin were mediated through UA lowering ${ }^{32}$.

Interestingly, post hoc analyses to determine the drivers of the relationship between reduction in UA by short-term fenofibrate and long-term cardiovascular outcomes revealed that the relationship was largely unaltered by further adjustment for baseline renal function, cardiovascular risk factors, smoking status and prior cardiovascular disease. Rather, the relationship appeared to have been driven by the strong trend towards benefit $(p=0.055)$ that females experience with every $0.1 \mathrm{mmol} / \mathrm{L}$ greater short-term reduction in UA levels conferring a 22\% lower cardiovascular risk over five years. In contrast, the relationship between short-term reduction in UA and cardiovascular risk was not present in males $(H R=1.0)$. Previous analyses of FIELD data have also noted more favourable lipoprotein improvements on fenofibrate in females than males ${ }^{33}$. Nevertheless, gender was not a statistically significant effect modifier of the relationship between UA reductions with short-term fenofibrate and cardiovascular risk ( $p$ for interaction effect $=0.10$ ). 
The current study needs to be interpreted in the context of several limitations. Firstly, there was a narrow spectrum of renal disease due to the FIELD trial excluding any participants with serum creatinine $>130 \mu \mathrm{mol} / \mathrm{L}$. Since fenofibrate-induced UAlowering is mediated through increased renal excretion of UA, exploration of current results across a wider range of renal function would be of clinical utility. Furthermore, as the study excluded patients with severe renal impairment, this may increase the probability of including more overproducers of UA, and therefore observing a clinical benefit with fenofibrate-mediated UA lowering ${ }^{34,35}$. Secondly, being a retrospective analysis, whether the relationship between UA and cardiovascular risk is causal or correlational could not be fully defined. However, the association between UA and baseline cardiovascular risk factors and on-study cardiovascular events, even after adjustment for multiple other risk factors, should prompt consideration of UA as a causal mechanism ${ }^{36}$. Thirdly, the current study analysed UA in plasma, which is less commonly used than serum in clinical practice, however, the analytical method using a Cobas enzymatic analyser has been validated for both plasma and serum UA analyses.

In conclusion, baseline UA levels and the changes therein after short-term fenofibrate use are both independent associates of subsequent cardiovascular events in patients with T2D. However, the UA lowering alone does not fully account for all of fenofibrate's cardiovascular benefits. 


\section{References}

1. Mooradian AD. Dyslipidemia in type 2 diabetes mellitus. Nat Clin Pract Endocrinol Metab. 2009;5(3):150-159.

2. Keech A, Simes RJ, Barter P, et al. Effects of long-term fenofibrate therapy on cardiovascular events in 9795 people with type 2 diabetes mellitus (the FIELD study): randomised controlled trial. Lancet. 2005;366(9500):1849-1861.

3. Noonan JE, Jenkins AJ, Ma JX, Keech AC, Wang JJ, Lamoureux EL. An update on the molecular actions of fenofibrate and its clinical effects on diabetic retinopathy and other microvascular end points in patients with diabetes. Diabetes. 2013;62(12):3968-3975.

4. Fulcher J, O'Connell R, Voysey M, et al. Efficacy and safety of LDL-lowering therapy among men and women: meta-analysis of individual data from 174,000 participants in 27 randomised trials. Lancet. 2015;385(9976):1397-1405.

5. Daskalopoulou SS, Tzovaras V, Mikhailidis DP, Elisaf M. Effect on serum uric acid levels of drugs prescribed for indications other than treating hyperuricaemia. Curr Pharm Des. 2005;11(32):4161-4175.

6. Waldman B, Ansquer JC, Sullivan DR, et al. Effect of fenofibrate on uric acid and gout in type 2 diabetes: a post-hoc analysis of the randomised, controlled FIELD study. Lancet Diabetes Endocrinol. 2018;6(4):310-318.

7. Fang J, Alderman MH. Serum uric acid and cardiovascular mortality the NHANES I epidemiologic follow-up study, 1971-1992. National Health and Nutrition Examination Survey. Jama. 2000;283(18):2404-2410.

8. Niskanen LK, Laaksonen DE, Nyyssonen $\mathrm{K}$, et al. Uric acid level as a risk factor for cardiovascular and all-cause mortality in middle-aged men: a prospective cohort study. Arch Intern Med. 2004;164(14):1546-1551.

9. Culleton BF, Larson MG, Kannel WB, Levy D. Serum uric acid and risk for cardiovascular disease and death: the Framingham Heart Study. Ann Intern Med. 1999;131(1):7-13.

10. Sakata K, Hashimoto T, Ueshima H, Okayama A. Absence of an association between serum uric acid and mortality from cardiovascular disease: NIPPON DATA 80, 19801994. National Integrated Projects for Prospective Observation of Noncommunicable Diseases and its Trend in the Aged. Eur J Epidemiol. 2001;17(5):461468.

11. The need for a large-scale trial of fibrate therapy in diabetes: the rationale and design of the Fenofibrate Intervention and Event Lowering in Diabetes (FIELD) study. [ISRCTN64783481]. Cardiovasc Diabetol. 2004;3:9.

12. Executive Summary of The Third Report of The National Cholesterol Education Program (NCEP) Expert Panel on Detection, Evaluation, And Treatment of High Blood Cholesterol In Adults (Adult Treatment Panel III). Jama. 2001;285(19):2486-2497. 
13. Yuan H, Yu C, Li X, et al. Serum Uric Acid Levels and Risk of Metabolic Syndrome: A Dose-Response Meta-Analysis of Prospective Studies. J Clin Endocrinol Metab. 2015;100(11):4198-4207.

14. De Cosmo S, Viazzi F, Pacilli A, et al. Serum Uric Acid and Risk of CKD in Type 2 Diabetes. Clin J Am Soc Nephrol. 2015;10(11):1921-1929.

15. Obermayr RP, Temml C, Gutjahr G, Knechtelsdorfer M, Oberbauer R, Klauser-Braun R. Elevated uric acid increases the risk for kidney disease. J Am Soc Nephrol. 2008;19(12):2407-2413.

16. Grayson PC, Kim SY, LaValley M, Choi HK. Hyperuricemia and incident hypertension: a systematic review and meta-analysis. Arthritis Care Res (Hoboken). 2011;63(1):102110.

17. Krishnan E, Choi HK, De Vera MA. Gout and the risk of type 2 diabetes among men with a high cardiovascular risk profile. Rheumatology. 2008;47(10):1567-1570.

18. Bhole V, Choi JW, Kim SW, de Vera M, Choi H. Serum uric acid levels and the risk of type 2 diabetes: a prospective study. Am J Med. 2010;123(10):957-961.

19. Dehghan A, van Hoek M, Sijbrands EJ, Hofman A, Witteman JC. High serum uric acid as a novel risk factor for type 2 diabetes. Diabetes Care. 2008;31(2):361-362.

20. Yoo TW, Sung KC, Shin HS, et al. Relationship between serum uric acid concentration and insulin resistance and metabolic syndrome. Circ J. 2005;69(8):928-933.

21. Chen LY, Zhu WH, Chen ZW, et al. Relationship between hyperuricemia and metabolic syndrome. J Zhejiang Univ Sci B. 2007;8(8):593-598.

22. Hamaguchi S, Furumoto T, Tsuchihashi-Makaya M, et al. Hyperuricemia predicts adverse outcomes in patients with heart failure. Int J Cardiol. 2011;151(2):143-147.

23. Holme I, Aastveit AH, Hammar N, Jungner I, Walldius G. Uric acid and risk of myocardial infarction, stroke and congestive heart failure in 417,734 men and women in the Apolipoprotein MOrtality RISk study (AMORIS). J Intern Med. 2009;266(6):558-570.

24. Filippatos GS, Ahmed MI, Gladden JD, et al. Hyperuricaemia, chronic kidney disease, and outcomes in heart failure: potential mechanistic insights from epidemiological data. Eur Heart J. 2011;32(6):712-720.

25. Krishnan E, Svendsen K, Neaton JD, Grandits G, Kuller LH. Long-term cardiovascular mortality among middle-aged men with gout. Arch Intern Med. 2008;168(10):11041110.

26. Ito $H, A$ be $M$, Mifune $M$, et al. Hyperuricemia is independently associated with coronary heart disease and renal dysfunction in patients with type 2 diabetes mellitus. PLoS One. 2011;6(11):e27817.

27. Zoppini G, Targher G, Negri C, et al. Elevated serum uric acid concentrations independently predict cardiovascular mortality in type 2 diabetic patients. Diabetes Care. 2009;32(9):1716-1720. 
28. Ong G, Davis WA, Davis TM. Serum uric acid does not predict cardiovascular or allcause mortality in type 2 diabetes: the Fremantle Diabetes Study. Diabetologia. 2010;53(7):1288-1294.

29. $\mathrm{Xu} \mathrm{Y,} \mathrm{Zhu} \mathrm{J,} \mathrm{Gao} \mathrm{L,} \mathrm{et} \mathrm{al.} \mathrm{Hyperuricemia} \mathrm{as} \mathrm{an} \mathrm{independent} \mathrm{predictor} \mathrm{of} \mathrm{vascular}$ complications and mortality in type 2 diabetes patients: a meta-analysis. PLoS One. 2013;8(10):e78206.

30. Juutilainen A, Kortelainen S, Lehto S, Ronnemaa T, Pyorala K, Laakso M. Gender difference in the impact of type 2 diabetes on coronary heart disease risk. Diabetes Care. 2004;27(12):2898-2904.

31. Krysiak R, Gdula-Dymek A, Bachowski R, Okopien B. Pleiotropic effects of atorvastatin and fenofibrate in metabolic syndrome and different types of prediabetes. Diabetes Care. 2010;33(10):2266-2270.

32. Athyros VG, Mikhailidis DP, Liberopoulos EN, et al. Effect of statin treatment on renal function and serum uric acid levels and their relation to vascular events in patients with coronary heart disease and metabolic syndrome: a subgroup analysis of the GREek Atorvastatin and Coronary heart disease Evaluation (GREACE) Study. Nephrol Dial Transplant. 2007;22(1):118-127.

33. d'Emden MC, Jenkins AJ, Li L, et al. Favourable effects of fenofibrate on lipids and cardiovascular disease in women with type 2 diabetes: results from the Fenofibrate Intervention and Event Lowering in Diabetes (FIELD) study. Diabetologia. 2014;57(11):2296-2303.

34. Borghi C, Desideri G. Urate-Lowering Drugs and Prevention of Cardiovascular Disease: The Emerging Role of Xanthine Oxidase Inhibition. Hypertension. 2016;67(3):496-498.

35. Ndrepepa G. Uric acid and cardiovascular disease. Clin Chim Acta. 2018;484:150-163.

36. Tsouli SG, Liberopoulos EN, Mikhailidis DP, Athyros VG, Elisaf MS. Elevated serum uric acid levels in metabolic syndrome: an active component or an innocent bystander? Metabolism. 2006;55(10):1293-1301. 


\section{Figure Legends}

Figure 1. Frequency histogram of baseline plasma uric acid ( $n=9622)$, divided into gender-specific tertiles with individual tertile ranges for both genders shown by bars above the histogram (male: $n=6042$; female: $n=3580$ ). * Uric acid treatment target in non-tophaceous gout $(0.36 \mathrm{mmol} / \mathrm{L})$ recommended by European League Against Rheumatism. The shaded red column represents the mean baseline uric acid $(0.32$ $\mathrm{mmol} / \mathrm{L})$.

Figure 2. Cardiovascular event by tertiles of baseline uric acid. Cumulative rate of total cardiovascular events over median 5 years follow-up presented next to figure legend.

Figure 3. A) Hazard ratio for long-term cardiovascular events on fenofibrate compared to control, stratified by absolute UA reduction during fenofibrate run-in. UA reduction was grouped as greater than or equal to median $(0.07 \mathrm{mmol} / \mathrm{L})$, or less than median, as represented by the top two bars. B) Hazard ratio for long-term cardiovascular events for every $0.1 \mathrm{mmol} / \mathrm{L}$ run-in reduction in plasma uric acid on fenofibrate (adjusted for baseline uric acid and long-term treatment allocation). The interval marks are 0.1-unit increments between HR 0.5-1, and 1-unit increments between HR 1-5. HR = hazard ratio. UA = uric acid 


\begin{tabular}{|c|c|c|c|c|}
\hline & $\begin{array}{l}\text { Lowest Tertile }(n=3248) \\
\text { Levels }\end{array}$ & $\begin{array}{l}\text { Middle Tertile }(n=3206) \\
\text { Levels }\end{array}$ & $\begin{array}{l}\text { Highest Tertile }(n=3168) \\
\text { Levels }\end{array}$ & $p$ value \\
\hline Gender, male (\%) & $2107(65)$ & $1941(61)$ & $1994(63)$ & 0.11 \\
\hline Ethnicity & & & & $<0.001$ \\
\hline Caucasian & $3049(94)$ & $2995(93)$ & $2883(91)$ & \\
\hline Asian & $56(2)$ & $43(1)$ & $42(1)$ & \\
\hline Others & $143(4)$ & $168(5)$ & $243(8)$ & \\
\hline Hypertension & $1655(51)$ & $1745(54)$ & $2042(64)$ & $<0.001$ \\
\hline Metabolic syndrome & 2549 (79) & $2745(86)$ & $2894(91)$ & $<0.001$ \\
\hline Smoking & & & & $<0.001$ \\
\hline Non-smoker & $1347(41)$ & $1322(41)$ & $1191(38)$ & \\
\hline Ex-smoker & $1535(47)$ & $1588(50)$ & $1732(55)$ & \\
\hline Current smoker & $366(11)$ & $296(9)$ & $245(8)$ & \\
\hline Diuretics & $277(8)$ & $392(12)$ & $780(25)$ & $<0.001$ \\
\hline \multicolumn{5}{|l|}{ Clinical characteristics } \\
\hline Systolic blood pressure, $\mathrm{mmHg}$ & $139(16)$ & $141(15)$ & $141(15)$ & $<0.001$ \\
\hline Diastolic blood pressure, $\mathrm{mmHg}$ & $81(8.6)$ & $82(8.4)$ & $82(8.5)$ & $<0.001$ \\
\hline
\end{tabular}


Pulse pressure, $\mathrm{mmHg}$

BMI, kg/m²

Laboratory data

Total cholesterol, $\mathrm{mmol} / \mathrm{L}$

Triglyceride, $\mathrm{mmol} / \mathrm{L}$

LDL-C, $\mathrm{mmol} / \mathrm{L}$

HDL-C, mmol/L

Non-HDL-C, mmol/L

Dyslipidaemia $^{\dagger}(\%)$

HbA1c, \%

eGFR, $\mathrm{mL} / \mathrm{min} / 1.73 \mathrm{~m}^{2}$

eGFR $<60 \mathrm{~mL} / \mathrm{min} / 1.73 \mathrm{~m}^{2}(\%)$

Albuminuria (\%)

Normal

Microalbuminuria ${ }^{\dagger \dagger}$

Macroalbuminuria ${ }^{\dagger \dagger}$

$58(13)$
$29(5.2)$

$5.0(0.69)$
$1.6(1.2-2.1)$
$3.1(0.63)$
$1.1(0.28)$
$3.9(0.67)$
$968(30)$
$7.3(6.4-8.4)$
$90(80-98)$
$81(2)$

$2462(76)$

$688(21)$

$94(3)$

$59(12)$
$31(5.3)$

$5.0(0.71)$
$1.8(1.4-2.3)$
$3.1(0.66)$
$1.1(0.25)$
$4.0(0.69)$
$1262(39)$
$6.8(6.1-7.7)$
$87(75-95)$
$146(5)$

$2428(76)$

$647(20)$

119 (4)
59 (12)

$32(5.8)$

0.006

$<0.001$

0.54

$<0.001$

$<0.001$

$<0.001$

$<0.001$

$<0.001$

$<0.001$

$<0.001$

$<0.001$

$<0.001$

Table 1. Baseline clinical and laboratory variables between baseline uric acid tertiles. Trend tests are used to derive all $p$ values. Continuous outcomes are reported as mean (SD) if normally distributed and median (IQR) if not normally distributed. eGFR = estimated glomerular filtration rate. HDL-C = high density lipoprotein cholesterol. LDL-C = low density lipoprotein cholesterol. ${ }^{\dagger}$ Dyslipidaemia was defined as low HDL-C (male: $<1.03 \mathrm{mmol} / \mathrm{L}$; female: $<1.29 \mathrm{mmol} / \mathrm{L}$ ) and high triglyceride $(\geq 1.7 \mathrm{mmol} / \mathrm{L})$.

${ }^{\dagger \dagger}$ Microalbuminuria was defined as urinary albumin-to-creatinine ratio 2.5-25 in male or 3.5-35 in female. ${ }^{\dagger \dagger}$ Macroalbuminuria was defined as urinary albumin-to-creatinine ratio $>25$ in male or $>35$ in female. 


\begin{tabular}{|c|c|c|c|c|}
\hline & Unadjusted & $p$ value & Adjusted & $p$ value \\
\hline Baseline uric acid (per $0.1 \mathrm{mmol} / \mathrm{L}$ ) & $1.21(1.13-1.29)$ & $<0.001$ & $1.09(1.01-1.18)$ & 0.020 \\
\hline Age (y) & & & $1.04(1.03-1.05)$ & $<0.001$ \\
\hline \multicolumn{5}{|l|}{ Gender } \\
\hline Male $^{\dagger}$ & & & 1 & \\
\hline Female & & & $0.55(0.48-0.63)$ & $<0.001$ \\
\hline \multicolumn{5}{|l|}{ Treatment allocation } \\
\hline Placebo $^{\dagger}$ & & & 1 & \\
\hline Fenofibrate & & & $0.87(0.78-0.97)$ & 0.015 \\
\hline Baseline BMI $\left(\mathrm{kg} / \mathrm{m}^{2}\right)$ & & & $1.00(0.99-1.02)$ & 0.49 \\
\hline \multicolumn{5}{|l|}{ Prior CVD } \\
\hline $\mathrm{No}^{\dagger}$ & & & 1 & \\
\hline Yes & & & $2.21(1.97-2.48)$ & $<0.001$ \\
\hline \multicolumn{5}{|l|}{ Smoking } \\
\hline Non-smoker ${ }^{\dagger}$ & & & 1 & \\
\hline Ex-smoker & & & $1.06(0.93-1.19)$ & 0.44 \\
\hline Current smoker & & & $1.53(1.27-1.85)$ & $<0.001$ \\
\hline Baseline systolic BP (per $10 \mathrm{mmHg}$ ) & & & 1.10 (1.06-1.14) & $<0.001$ \\
\hline HbA1c (per $1 \%$ ) & & & 1.13 (1.08-1.18) & $<0.001$ \\
\hline Baseline triglycerides (per $1 \mathrm{mmol} / \mathrm{L}$ ) & & & $1.03(0.97-1.10)$ & 0.33 \\
\hline Baseline non-HDL-C (per $1 \mathrm{mmol} / \mathrm{L})$ & & & 1.28 (1.17-1.39) & $<0.001$ \\
\hline \multicolumn{5}{|l|}{ Composite eGFR/albuminuria } \\
\hline eGFR $\geq 60$, no albuminuria & & & 1 & \\
\hline eGFR $<60$, no albuminuria & & & $1.27(0.97-1.67)$ & 0.078 \\
\hline eGFR $\geq 60$, albuminuria & & & $1.28(1.13-1.46)$ & $<0.001$ \\
\hline
\end{tabular}


Table 2. Unadjusted and adjusted (for each listed variable) Cox proportional models of baseline uric acid as a predictor of cardiovascular events (HR for every $0.1 \mathrm{mmol} / \mathrm{L}$ higher baseline uric acid). ${ }^{\dagger}$ Reference group. * $p<0.05$ for continuous variables or $p<0.05$ compared to reference group for categorical variables. 95\% confidence interval presented in parentheses after each hazard ratio. $\mathrm{BMI}=$ body mass index. $\mathrm{BP}=$ blood pressure. $\mathrm{CVD}=$ cardiovascular disease. Non-HDL-C $=$ Non-high-density lipoprotein cholesterol. 